\title{
Corrigendum: Identifying signatures of photothermal current in a double-gated semiconducting nanotube
}

\author{
G. Buchs, S. Bagiante \& G.A. Steele
}

Nature Communications 5:4987 doi: 10.1038/ncomms5987 (2014); Published 19 Sep 2014; Updated 23 Jan 2015

The original version of this Article contained an error in the spelling of 'Identifying' in the title of the paper. This has now been corrected in both the PDF and HTML versions of the Article. 